\title{
Simulations of thermophoretic nanoswimmers
}

\author{
Mingcheng Yang* and Marisol Ripoll ${ }^{\dagger}$ \\ Theoretical Soft-Matter and Biophysics, Institute of Complex Systems, Forschungszentrum Jülich, D-52425 Jülich, Germany
}

(Received 5 August 2011; revised manuscript received 5 September 2011; published 6 December 2011)

\begin{abstract}
We consider a nanodimer in solution with asymmetric thermal properties that shows self-propelled motion. One monomer of the nanodimer can be heated to a fixed temperature producing a radially symmetric temperature gradient. The thermophoretic properties of the second monomer produce then a propulsion against or toward the heated particle, such that the nanodimer becomes a puller or pusher nanoswimmer. We combine our simulation measurements with a theoretical analysis that satisfactorily characterizes the self-propelled velocity with the temperature gradient, and the thermophoretic properties of the bead.
\end{abstract}

DOI: 10.1103/PhysRevE.84.061401

PACS number(s): 82.70.-y, 66.10.cd, 87.17.Jj, 05.70.Ln

\section{INTRODUCTION}

Molecular motors are ubiquitous in biology, with examples ranging from motor proteins moving along filaments [1], to swimming bacteria [2]. In such systems self-propulsion is mostly achieved by using chemical energy released from adenosine triphosphate (ATP) hydrolysis [3]. Recently, synthetic microscale and nanoscale motors have attracted considerable attention due to their potential practical applications and related theoretical open questions $[4,5]$. A relatively simple and effective strategy to design artificial nanomotors has already been found in employing phoretic effects. Phoresis refers to the drift motion of a suspended particle produced by the mechanical force that arises from an inhomogeneous fluid environment. Such inhomogeneities can be gradients of electric potential (electrophoresis), concentration (diffusiophoresis), or temperature (thermophoresis) [6]. These gradients are frequently a consequence of external constrains, but interestingly, in the case that one particle is able to produce a local gradient field by itself, self-propulsion may occur. The theoretical basis of this phenomenon has been discussed by different authors [6,7]. Following this line and by means of simulations [8] and experiments [9,10], a chemical reaction catalyzed asymmetrically on a particle's surface has shown to translate into a diffusiophoresis motor. More recently, a Janus particle has been shown to display self-propelled motion due to thermophoresis [11]. In their experiments, Jiang et al. employ a half-metal coated colloidal sphere and heat it with a defocused laser. The higher heat absorption of the metal side produces a temperature gradient on the nonmetal side, which translates into a self-propelled motion.

In this work we perform computer simulations of nanodimers that swim due to thermophoresis at low Reynolds numbers. Two strongly bonded monomers are immersed in a hydrodynamic solvent as depicted in Fig. 1. The heated bead $h$ can have a temperature higher than the surrounding fluid. This accounts for a monomer of material as gold that absorbs heat, for example, from a laser. If the average temperature $\bar{T}$ of the system is kept constant, the surrounding solvent will sustain a steady temperature gradient with radial symmetry. The nonheated bead can generate a thrust due to

\footnotetext{
*m.yang@fz-juelich.de

${ }^{\dagger}$ m.ripoll@fz-juelich.de
}

thermophoresis, which will translate into a directed motion of the nanodimer along the bond direction. We will therefore refer to the nonheated bead as the propelling monomer $p$. In the case where $p$ bead is thermophilic, it will tend to go to higher temperatures, and the nanodimer will behave as a pusher. Reciprocally, in the case where the $p$ bead is thermophobic, the nanodimer will behave as a puller [12]. The propulsion velocity $v_{p}$ is measured in our simulations and theoretically satisfactorily explained. Our system nicely mimics the recent experiments by Jiang et al. [11] with a simpler geometry of the temperature profile.

\section{SIMULATION MODEL}

Simulations are performed with a hybrid scheme. The solvent is described by a particle-based mesoscopic simulation technique known as multiparticle collision dynamic (MPC) [13], while monomers and their interactions with the solvent are simulated by standard molecular dynamics (MD). MPC consists of alternating streaming and collision steps. In the streaming step, the solvent particles of mass $m$ move ballistically for a time $h$. In the collision step, particles are sorted into a cubic lattice with cells of size $a$, and their velocities relative to the center-of-mass velocity of each cell are rotated around a random axis by an angle $\alpha$. In the collisions, mass, momentum, and energy are locally conserved. This allows the algorithm to properly capture hydrodynamic interactions, thermal fluctuations, and the sustainability of temperature inhomogeneities [14]. MPC has already been shown to be successful in the simulation of other self-propelled particles $[8,15,16]$. A detailed description of the method can be found, e.g., in Refs. [17-19]. We employ the standard MPC parameters $\alpha=130^{\circ}, h=0.1$, and the mean number of solvent particles per cell $\rho=10$. Simulation units are chosen to be $m=1, a=1$, and $k_{B} \bar{T}=1$, where $k_{B}$ is the Boltzmann constant and $\bar{T}$ is the average system temperature. Time and velocity are consequently scaled with $\left(m a^{2} / k_{B} \bar{T}\right)^{1 / 2}$ and $\left(k_{B} \bar{T} / m\right)^{1 / 2}$, respectively.

The solvent particles interact with the nanodimer beads through Lennard-Jones (LJ) type potentials. They can be attractive or repulsive, with the general form

$$
U_{k}(r)=4 \epsilon\left[\left(\frac{\sigma}{r}\right)^{2 n_{k}}-\left(\frac{\sigma}{r}\right)^{n_{k}}\right]+C_{k}, \quad r \leqslant r_{c}
$$




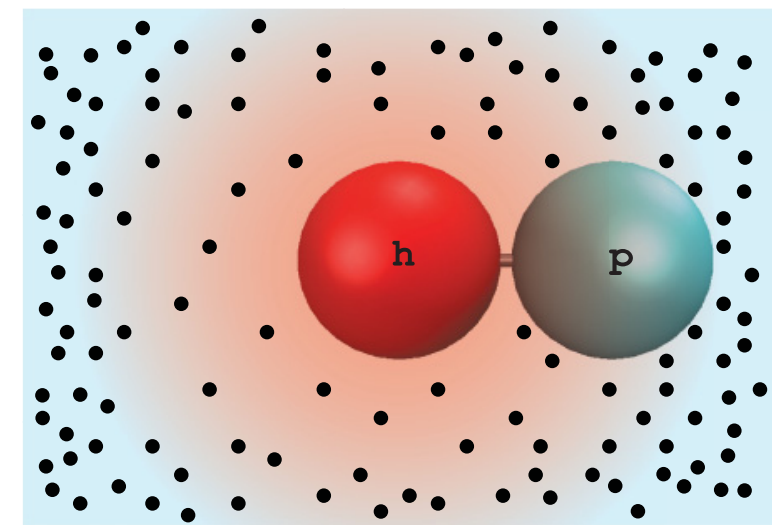

FIG. 1. (Color online) Schematic diagram of the simulated nanodimer solution. The $h$ monomer can be heated to a fixed temperature and it is strongly linked to a second monomer $p$. The system has then a radially symmetric temperature gradient.

where $k$ may be $h$ or $p$. The potential intensity is always chosen as $\epsilon=k_{B} \bar{T}$, and the monomer's radius as $\sigma=2.5 a . n_{k}$ is a positive integer that describes the stiffness of the potential, and $r_{c}$ is potential cutoff radius. The attractive LJ potential is obtained with $C_{k}=0$, and the repulsive with $C_{k}=\epsilon$. In our simulations, $U_{h}$ is fixed to be strongly repulsive with $C_{h}=\epsilon$ and $n_{h}=24$, while various choices of $U_{p}$ have been made. Both bead masses are $M=650 \mathrm{~m}$, such that the beads are neutrally buoyant. A strong harmonic potential is used to fix the interbead separation to $l=5.5 a$.

In order to fix the temperature of the $h$ bead, we rescale the thermal energy of the solvent particles closer than $\sigma+0.2 a$ to the center of the bead to a value $\frac{3}{2} k_{B} T_{h}$. This means that only a small layer $(\simeq 0.08 \sigma)$ around the $h$ bead is affected by the rescaling. The inserted energy is drained from the system by fixing the mean temperature $\bar{T}$ of the system. In experiments, the thermalization is performed at the system boundaries, which is related to a different energy flux mechanism. Nonetheless, when the system is large enough, and considering especially the nanodimer neighborhood, the differences are expected to be negligible.

The temperature profile around the $h$ bead, given a fixed $T_{h}$ temperature, will be time independent if the characteristic time of the thermal energy propagation $\tau_{\chi}$ is largely separated from the bead diffusion time $\tau_{D}$. This occurs in experimental systems, where a hot Brownian particle carries with it a radially symmetric temperature distribution [20]. These two quantities can be explicitly written as $\tau_{\chi} \sim \sigma^{2} / \chi$ with $\chi$ the heat diffusivity, and $\tau_{D} \sim \sigma^{2} / D$, with the self-diffusion coefficient $D=k_{B} \bar{T} / 4 \pi \eta \sigma$ (slip boundary) and $\eta$ the shear viscosity. Kinetic theory of MPC [21] provides a very accurate approximation of $\eta$ and $\chi$ in terms of the employed parameters, which allows us to calculate that $\tau_{\chi} / \tau_{D} \sim 10^{-2}$ for our simulation parameters. Hence this important time-scale separation can be properly taken into account by our model when simulating the thermal energy-driven motor.

The spherical symmetry of the temperature distribution around the $h$ bead, together with the energy conservation, and the Fourier's law implies a temperature field $T(r)=$ $A_{1} / r+A_{2}$ with $r$ the distance to the $h$-bead center. The constant factors $A_{1}$ and $A_{2}$ are determined given the imposed

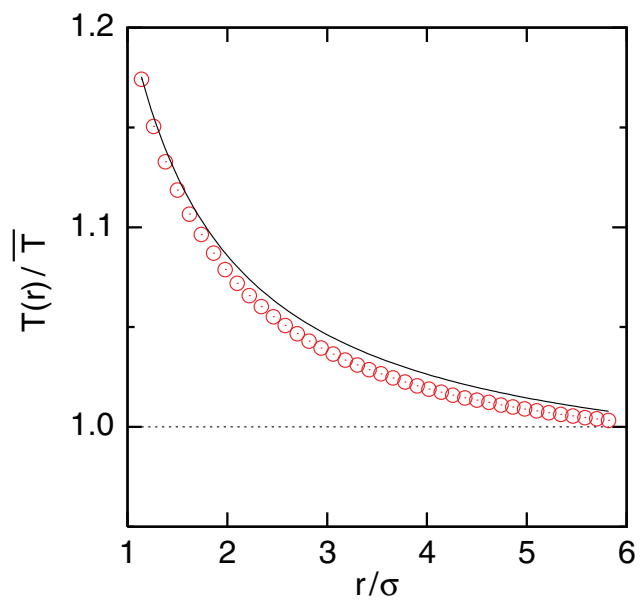

FIG. 2. (Color online) Temperature at a distance $r$ from the center of the $h$ bead in units of the bead radius $\sigma$, with $T_{h}=1.2 \bar{T}$. Symbols correspond to the simulation measurements and the line to the analytical prediction.

temperatures $T_{h}$ and $\bar{T}$. In Fig. 2 a typical temperature profile around the $h$ bead as measured from the simulations is plotted together with the predicted profile. The small deviation is due to the approximations made in the analytical calculation.

\section{RESULTS AND DISCUSSION}

The steady state temperature gradient leads to a thermophoretic force on the $p$ bead that will tend to move along the nanodimer bond direction. The resulting self-propelled velocity $v_{p}$ can then be positive (towards the heated particle), or negative (against the heated particle), depending on the sign of the Soret coefficient of the $p$ bead. During its motion the nanodimer can freely rotate, which implies that the directed motion will change orientation, which will effectively give rise to an enhanced diffusive behavior $[9,11]$. Figure 3 depicts the trajectories of the nanodimer center of mass projected on the $x y$ plane. It is found that the self-propelled nanodimer can explore a larger area with increasing thermophoretic force, which suggests that the self-propelled nanodimer has indeed a larger effective diffusion coefficient.

To characterize the self-propelled velocity $v_{p}$, the nanodimer center-of mass-velocity $\mathbf{v}_{c m}$ is projected in the bond (a)

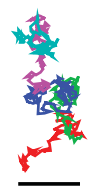

(b)

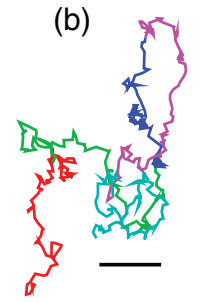

(c)

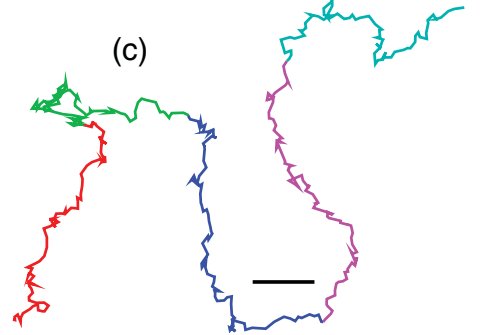

FIG. 3. (Color online) Trajectories of the center of mass of the nanodimer projected on $x y$ plane with increasing self-propelled velocity for a fixed total time. Reference bars indicate the nanodimer size. (a) No temperature gradient and $v_{p}=0$. (b), (c) $T_{h}=1.2 \bar{T}$ with increasing $v_{p}$. (b) Repulsive $U_{p}\left(C_{P}=\epsilon, n_{p}=6\right)$. (c) Attractive $U_{p}$ $\left(C_{P}=0, n_{p}=24\right)$. 


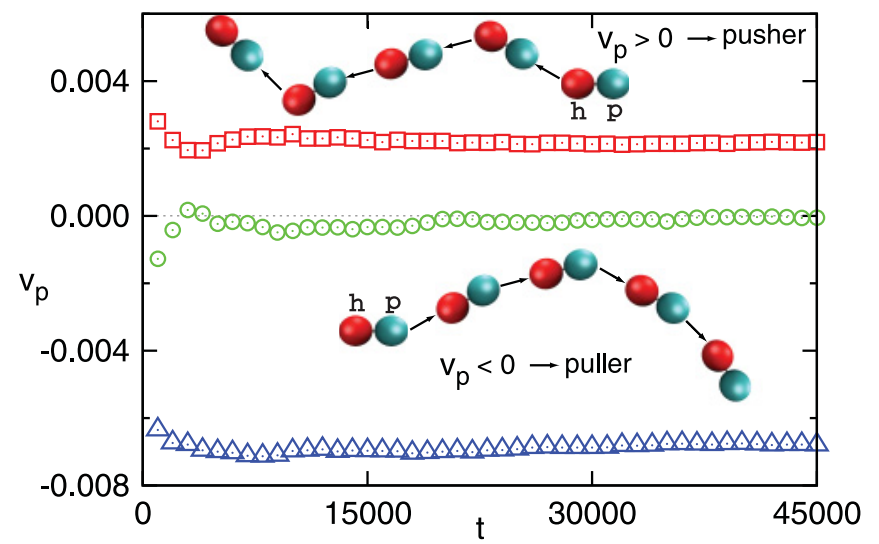

FIG. 4. (Color online) Nanodimer self-propelled velocity along the bond direction as a function of time as averaging parameter. Simulation parameters correspond to those in Fig. 3: circles to (a), squares to (b), and triangles to (c). Swimming characteristic behaviors are illustrated in the insets [12].

direction $\mathbf{n}=\mathbf{r}_{h p} /\left|\mathbf{r}_{h p}\right|$, such that $v_{p}=\left\langle\mathbf{v}_{c m} \cdot \mathbf{n}\right\rangle$. In Fig. $4, v_{p}$ is averaged over a long time and clearly determined values are shown. The employed repulsive interaction is known to display a negative $S_{T}$ [14] (this is thermophilic), which agrees with the observed pusher behavior given by $v_{p}=0.0022$. Meanwhile the attractive interaction has a positive $S_{T}$ (thermophobic), which agrees with the observed puller behavior given by $v_{p}=$ -0.0068 . As a reference, results in the absence temperature gradient are presented, where a vanishing $v_{p}$ is obtained. The Reynolds number $\operatorname{Re}=v_{p} \sigma \rho / \eta$ can then be evaluated to be $\operatorname{Re} \in\left(10^{-2}, 10^{-1)}\right.$. This means that the self-propelled nanodimer operates in the environment of low Reynolds number [22].

In order to gain deeper insight into the phenomenon, we now explain the measurements of the self-propelled velocity in terms of the passive thermophoresis. It is well accepted $[23,24]$ that the presence of a temperature gradient can induce a thermophoretic force $f_{T}$ on a colloidal particle suspended in a fluid,

$$
f_{T}=-\alpha_{T} \nabla k_{B} T \text {. }
$$

The proportionality factor $\alpha_{T}$ is the thermal diffusion factor of the particle related to the Soret coefficient $S_{T}$ by $\alpha_{T}=$ $T S_{T}$. The presence of a thermophoretic force will then induce a directed velocity $v_{p}=\mu_{p} f_{T}$ such that the related viscous force balances the thermophoretic force. Similar to $v_{p}, \mu_{p}$ corresponds to the mobility along the bond direction. Note that there is no thermophoretic force exerted on the $h$ bead since its surface is at constant temperature. The self-propelled velocity can then be expressed as

$$
v_{p}=-\mu_{p} \alpha_{T} \nabla k_{B} T .
$$

The validity of this relation can be checked, since the involved quantities can be independently computed.

In order to quantify $\mu_{p}$, we assume the Einstein relation $D_{p}=\mu_{p} k_{B} T$ with the related self-diffusion coefficient $D_{p}$, which can be characterized by measuring the mean-squared displacement (MSD) of the nanodimer center of mass along the bond direction. The instantaneous displacement along

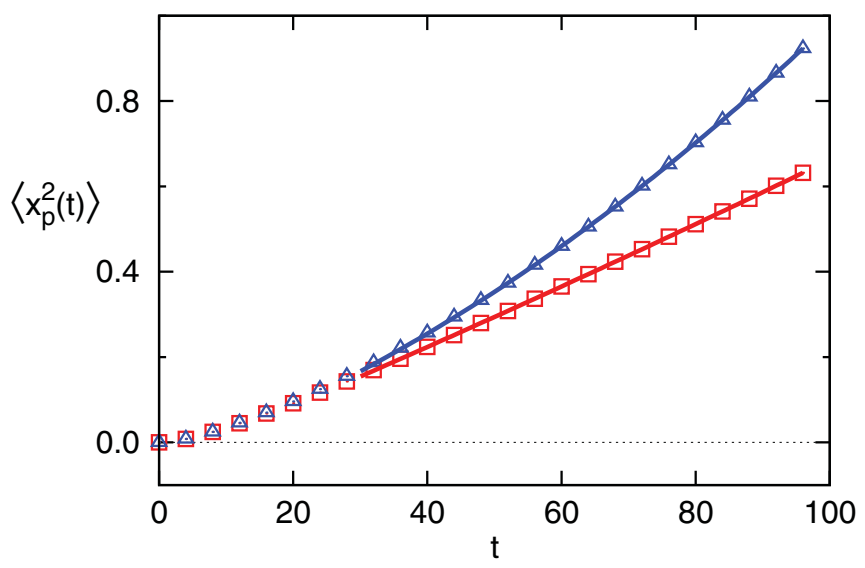

FIG. 5. (Color online) MSD of the nanodimer center of mass along the bond direction vs time. Simulation parameters are those of Fig. 4. Lines corresponds to fits with Eq. (4).

bond direction at a certain time $t_{i}$ in a differential time $\delta t$ can be expressed as $\delta x_{p}\left(t_{i}\right)=\left[\mathbf{r}_{c m}\left(t_{i}+\delta t\right)-\mathbf{r}_{c m}\left(t_{i}\right)\right] \cdot \mathbf{n}\left(t_{i}\right)$. The differential time should be small enough to consider $\mathbf{n}\left(t_{i}\right)$ constant. With this definition, the average displacement is related to the self-propelled velocity by $\left\langle\delta x_{p}\left(t_{i}\right)\right\rangle=v_{p} \delta t$. Consequently, the time dependent displacement along the bond direction can be calculated as $x_{p}(t)=\sum_{t_{i}=0}^{t} \delta x_{p}\left(t_{i}\right)$. When $v_{p}$ is considered, the MSD corresponds to a one-dimensional Brownian motion with $\left\langle\left(x_{p}(t)-\left\langle x_{p}(t)\right\rangle\right)^{2}\right\rangle=2 D_{p} t$. The MSD of the nanodimer center of mass along the bond direction can then be directly expressed as

$$
\left\langle x_{p}(t)^{2}\right\rangle=2 D_{p} t+v_{p}^{2} t^{2} .
$$

The MSD as a function of time is shown in Fig. 5 for two different potentials. At very small times, an initial purely quadratic regime due to inertial effects is observed. For times larger than the Brownian time, the diffusive behavior coexists with the presence of the self-propelled velocity as stated in Eq. (4). A fit to the data allows us to determine $D_{p}$ and $v_{p}$ with good accuracy, which for the displayed example are $D_{p}=0.0033$ and $v_{p}=0.0023$ (repulsive), and $D_{p}=0.0028$ and $v_{p}=-0.0068$ (attractive), respectively. Note that the values of $v_{p}$ nicely agree with the direct measurements shown in Fig. 4.

The quantities $\alpha_{T}$ and $S_{T}$ have been extensively measured from experiments and simulations [23-29] for various systems. MD simulations with single colloidal particles have been performed by Galliéro and Volz [28]. For the particular model and potentials chosen in this work we employ the values obtained from simulations by Lüsebrink [14] for a single colloid particle in a linear temperature gradient. It is known that MPC solvent has an ideal gas equation of state, however this does not influence the existence of the thermophoresis, and consequently of the self-propelled behavior treated in this work. In Fig. 6, direct measurements of $v_{p}$ are presented for different temperature gradients and potential interactions (different $\alpha_{T}$ ). The temperature gradient has been locally estimated at the center of the $p$ bead. Simultaneously, the values of $v_{p}$ indirectly obtained by computing the righthand side of Eq. (3) are presented for comparison. The agreement is satisfactory, though the indirect evaluated $v_{p}$ 

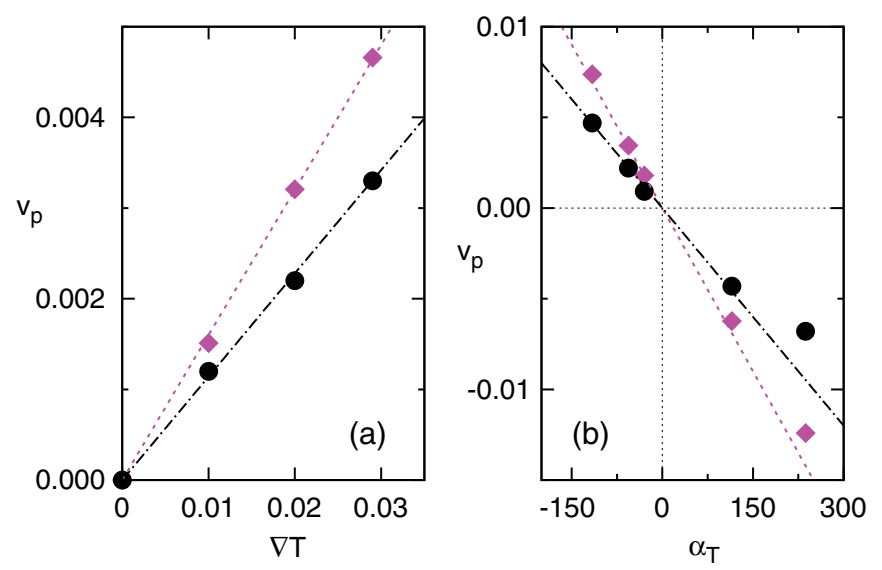

FIG. 6. (Color online) Self-propelled velocity as a function of the temperature gradient in (a) and of the thermal diffusion factor in (b). Bullets correspond to direct simulation results, diamonds to the evaluation of Eq. (3), and lines to linear fits. (a) The $p$ bead has a repulsive $U_{p}\left(C_{P}=\epsilon, n_{p}=6\right)$. From left to right, the points correspond to $T_{h}=\bar{T}, 1.1 \bar{T}, 1.2 \bar{T}, 1.3 \bar{T}$. (b) $T_{h}=1.2 \bar{T}$ is fixed and $U_{p}$ potentials are, from left to right, $\left(C_{p}=\epsilon, n_{p}=3\right),\left(C_{p}=\epsilon, n_{p}=\right.$ 6), $\left(C_{p}=\epsilon, n_{p}=12\right),\left(C_{p}=0, n_{p}=48\right)$, and $\left(C_{p}=0, n_{p}=24\right)$.

values systematically overestimate the directly measured ones.

The overestimation of $v_{p}$ obtained with the right-hand side of Eq. (3) can be attributed to several factors. (i) The employed values of $\alpha_{T}$ are obtained from single-particle simulations. In the nanodimer environment, the existence of the $h$ bead and the radial temperature gradient will have certain influence in the distribution of the solvent near the $p$ bead, and therefore in $\alpha_{T}$. (ii) The temperature gradient generated by the $h$ bead near the $p$ bead is, as shown in Fig. 2, rather large and decreases rapidly with the distance. Thus the local linear temperature gradient at the center of the $p$ bead is just an approximation. (iii) The Einstein relation assumed to calculate $\mu_{p}$ is not necessarily valid for a system far beyond equilibrium [20,30]. (iv) The temperature dependence of $\alpha_{T}$ [31] has not been considered in our estimations. (v) Equation (2) is based on the linear response theory, which is only the lowest-order approximation under large temperature gradients as those presented here. The clear linear behavior observed in Fig. 6 indicates that the main contributions might be in the determination of $\alpha_{T}, \nabla T$, and $\mu_{p}$ as indicated in (i)-(iv). Nevertheless, the relative importance of the different contributions should be further investigated. Reciprocally, in systems where a better agreement can be achieved, the self-propelled velocity could be employed to determine $\alpha_{T}[11,32]$.

\section{CONCLUSION}

In summary, we present a simulation model of the swimming motion caused by thermophoresis that can be theoretically well supported. The required local temperature gradient can be experimentally created by inhomogeneous absorption of external light [11] or by internally released heat from chemical reactions. The directed motion is achieved by the transformation of thermal energy of the nonequilibrium fluid environment into kinetic energy of the nanodimer. Thermophoresis appears then as a promising mechanism to design synthetic nanomachines.

\section{ACKNOWLEDGMENTS}

We want to thank D. Lüsebrink for discussions and the availability of his results prior to publication. We acknowledge interesting discussions with H. R. Jiang.
[1] J. Howard, Mechanics of Motor Proteins and the Cytoskeleton (Sinauer, NewYork, 2000).

[2] H. Berg, E. coli in Motion (Springer, New York, 2003).

[3] F. Jülicher, A. Ajdari, and J. Prost, Rev. Mod. Phys. 69, 1269 (1997).

[4] W. F. Paxton, K. C. Kistler, C. C. Olmeda, A. Sen, S. K. S. Angelo, Y. Cao, T. E. Mallouk, P. E. Lammert, and V. H. Crespi, J. Am. Chem. Soc. 126, 13424 (2004).

[5] R. Dreyfus, J. Baudry, M. L. Roper, M. Fermigier, H. A. Stone, and J. Bibette, Nature (London) 437, 862 (2005).

[6] J. L. Anderson, Annu. Rev. Fluid Mech. 21, 61 (1989).

[7] R. Golestanian, T. B. Liverpool, and A. Ajdari, Phys. Rev. Lett. 94, 220801 (2005). R. Golestanian, T. B. Liverpool, and A. Ajdari, New J. Phys. 9, 126 (2007).

[8] G. Rückner and R. Kapral, Phys. Rev. Lett. 98, 150603 (2007).

[9] J. R. Howse, R. A. L. Jones, A. J. Ryan, T. Gough, R. Vafabakhsh, and R. Golestanian, Phys. Rev. Lett. 99, 048102 (2007).

[10] L. F. Valadares, Y. G. Tao, N. S. Zacharia, V. Kitaev, F. Galembeck, R. Kapral, and G. A. Ozin, Small 6, 565 (2010).

[11] H. R. Jiang, N. Yoshinaga, and M. Sano, Phys. Rev. Lett. 105, 268302 (2010)
[12] See Supplemental Material at http://link.aps.org/supplemental/ 10.1103/PhysRevE.84.061401 for movies illustrating the swimming behavior as a puller or a pusher.

[13] A. Malevanets and R. Kapral, J. Chem. Phys. 110, 8605 (1999); 112, 7260 (2000).

[14] D. Lüsebrink, Ph.D thesis, University of Cologne, Cologne.

[15] M. T. Downton and H. Stark, J. Phys.: Condens. Matter 21, 204101 (2009).

[16] I. O. Götze and G. Gompper, Phys. Rev. E 82, 041921 (2010).

[17] M. Ripoll, K. Mussawisade, R. G. Winkler, and G. Gompper, Phys. Rev. E 72, 016701 (2005).

[18] G. Gompper, T. Ihle, D. M. Kroll, and R. G. Winkler, Adv. Polym. Sci. 221, 1 (2009).

[19] R. Kapral, Adv. Chem. Phys. 140, 89 (2008).

[20] D. Rings, R. Schachoff, M. Selmke, F. Cichos, and K. Kroy, Phys. Rev. Lett. 105, 090604 (2010).

[21] E. Tüzel, T. Ihle, and D. M. Kroll, Phys. Rev. E 74, 056702 (2006).

[22] E. M. Purcell, Am. J. Phys. 45, 3 (1977).

[23] A. Würger, Rep. Prog. Phys. 73, 126601 (2010). 
[24] R. Piazza and A. Parola, J. Phys.: Condens. Matter 20, 153102 (2008).

[25] S. Wiegand, J. Phys.: Condens. Matter 16, R357 (2004).

[26] S. Duhr and D. Braun, Proc. Natl. Acad. Sci. 103, 19678 (2006).

[27] M. Zhang and F. Müller-Plathe, J. Chem. Phys. 125, 124903 (2006).

[28] G. Galliéro and S. Volz, J. Chem. Phys. 128, 064505 (2008).
[29] D. Stadelmaier and W. Köhler, Macromolecules 41, 6205 (2008).

[30] V. Blickle, T. Speck, C. Lutz, U. Seifert, and C. Bechinger, Phys. Rev. Lett. 98, 210601 (2007).

[31] M. Braibanti, D. Vigolo, and R. Piazza, Phys. Rev. Lett. 100, 108303 (2008).

[32] R. Golestanian, Physics 3, 108 (2010). 